\title{
Meta-Analysis of Anxiety as a Risk Factor for Cardiovascular Disease
}

Connor A Emdin $\mathrm{HBSc}^{1}$, Ayodele Odutayo MD MSc ${ }^{2}$ Christopher X Wong MB ChB $\mathrm{PhD}^{3}$, Jenny Tran MB ChB ${ }^{4}$, Allan J Hsiao MPhil ${ }^{5}$, Benjamin HM Hunn MB ChB ${ }^{6}$

${ }^{1}$ St John’s College, University of Oxford, Oxford, United Kingdom

${ }^{2}$ Centre for Statistics in Medicine, Nuffield Department of Orthopaedics, Rheumatology and Musculoskeletal Sciences, University of Oxford, Oxford, United Kingdom

${ }^{3}$ Nuffield Department of Population Health, University of Oxford, Oxford, United Kingdom; and Centre for Heart Rhythm Disorders, University of Adelaide and Royal Adelaide Hospital, South Australian Health and Medical Research Institute

${ }^{4}$ Green Templeton College, University of Oxford, Oxford, United Kingdom

${ }^{5}$ Department of Economics, Massachusetts Institute of Technology

${ }^{6}$ Department of Physiology, Anatomy and Genetics, University of Oxford, Oxford, United Kingdom and School of Medicine, University of Tasmania, Hobart, Australia

\section{Correspondence to:}

Connor Emdin

St John's College,

34 Broad Street, Oxford OX1 3DB, United Kingdom

Tel: +447917507906; fax: +441865607202;

e-mail: connor.emdin@sjc.ox.ac.uk

Word Count: 2213 


\section{Abstract}

Whether anxiety is a risk factor for a range of cardiovascular diseases is unclear. We aimed to determine the association between anxiety and a range of cardiovascular diseases. MEDLINE and EMBASE were searched for cohort studies that included participants with and without anxiety, including individuals with anxiety, worry, posttraumatic stress disorder, phobic anxiety and panic disorder. We examined the association of anxiety with cardiovascular mortality, major cardiovascular events (defined as the composite of cardiovascular death, stroke, coronary heart disease and heart failure), stroke, coronary heart disease, heart failure and atrial fibrillation. We identified 46 cohort studies containing 2017276 participants and 222253 individuals with anxiety. Anxiety was associated with a significantly elevated risk of cardiovascular mortality (RR 1.41 CI 1.13, 1.76), coronary heart disease (RR $1.41 \mathrm{CI}$ 1.23, 1.61), stroke (RR 1.71 CI 1.18, 2.50) and heart failure (RR 1.35 CI 1.11, 1.64). Anxiety was not significantly associated with major cardiovascular events or atrial fibrillation, although confidence intervals were wide. Phobic anxiety was associated with a higher risk of coronary heart disease than other anxiety disorders and PTSD was associated with a higher risk of stroke. Results were broadly consistent in sensitivity analyses. Anxiety disorders are associated with an elevated risk of a range of different cardiovascular events, including stroke, coronary heart disease, heart failure and cardiovascular death. Whether these associations are causal is unclear.

Key Words: Psychosocial factors, Anxiety, Cardiovascular disease, Coronary heart disease 


\section{Introduction}

Despite the substantial evidence that depression and general psychological distress are associated with incident cardiovascular disease, the association between anxiety and cardiovascular disease is less clear. Previous results in studies conducted in cardiovascular disease populations, such as heart failure, have shown that anxiety is associated with a higher risk of adverse outcomes. ${ }^{1}$ However, these results may be due to reverse causality, that is, more severe cardiovascular disease causing greater anxiety rather than anxiety causing adverse outcomes. In a previous meta-analysis of 20 cohort studies, anxiety disorders were associated with a $26 \%$ higher risk of coronary heart disease. ${ }^{2}$ However, prior studies have provided conflicting results on whether anxiety disorders are associated with risk of stroke ${ }^{3}$, heart failure ${ }^{4}$ or cardiovascular mortality. ${ }^{5,6}$ Contribution of anxiety disorders to the development of cardiovascular disease would have substantial implications for the estimation of the global and regional burden of anxiety disorders, for prevention and treatment of anxiety disorders and for future research into the relationship between anxiety disorders and cardiovascular disease. We therefore aimed to conduct a comprehensive meta-analysis on the association between anxiety and incident cardiovascular disease and death.

\section{Methods}

Cohort studies or case-control studies nested within cohort studies were eligible for inclusion. Studies were required to contain adults with and without anxiety and were required to have a minimum of three months follow-up. Anxiety was defined as anxiety symptoms, generalized anxiety disorder (GAD), panic, phobia, posttraumatic stress disorder (PTSD), and worry, consistent with prior studies. ${ }^{2}$ Only studies that were conducted in a general population were eligible for inclusion; studies conducted on specific populations (e.g. type 2 diabetes, myocardial infarction) were 
excluded. We restricted our analysis to studies conducted in a general population to minimize the risk of reverse causality, that is, cardiovascular disease causing anxiety symptoms, and to ensure that our results were broadly applicable. Eligible studies also reported a measure of relative risk for at least one of the following outcomes: cardiovascular mortality, major cardiovascular events (defined as a composite of cardiovascular death, stroke, coronary heart disease and incident heart failure), stroke, coronary heart disease (coronary heart disease death, including sudden cardiac death, and non-fatal myocardial infarction), heart failure, atrial fibrillation and chronic kidney disease. No language restrictions were applied.

MEDLINE and EMBASE were searched from inception (1966) to July 2015 by an experienced research librarian. The following search terms were used for anxiety: “anxiety or post traumatic stress disorder or tension or anxiety symptoms or anxiety disorder or panic or panic attacks or phobic anxiety or phobia or worry”. 2 Bibliographic review of included studies was also conducted to identify potentially eligible studies. Two researchers screened all abstracts identified in the search in duplicate, excluding those that did not meet eligibility criteria. Following this screen, full texts of eligible studies were again assessed in duplicate by two separate researchers.

Data was extracted using a standardized form in duplicate. General study characteristics, including the population under study, number of participants with and without anxiety, duration of follow-up, mean age, number of men and number of participants with cardiovascular disease at baseline, were extracted. Maximally adjusted measures of relative risk and associated 95\% confidence intervals for outcomes of interest (cardiovascular mortality, major cardiovascular events, stroke, coronary heart disease, heart failure, atrial fibrillation and chronic kidney disease) were extracted, as well as the published covariates that investigators included in the 
regression model. Studies were then categorized as unadjusted, minimally adjusted or adequately adjusted, as previously performed. Unadjusted studies did not adjust for any potential confounders and were excluded from analysis. Minimally adjusted studies adjusted for age and were excluded in a sensitivity analysis. Adequately adjusted studies adjusted for age, sex and at least two established cardiovascular risk factors (blood pressure, cholesterol, smoking status, body mass index and diabetes). For studies that reported multiple anxiety categories (e.g. high symptoms of anxiety, moderate symptoms of anxiety, no anxiety symptoms), the relative risk for the most severe category was used. For studies that reported risk of cardiovascular disease per standard deviation of anxiety symptoms or by quantile (e.g. top fourth relative to the bottom fourth of anxiety symptoms), a normal distribution was assumed and the measure of relative risk standardized to correspond to the top $13.5 \%$ of the study population compared to the bottom $86.5 \%$ of the study population $(13.5 \%$ being the mean proportion of the population with anxiety among included studies). Risk of bias in included studies was assessed using the Newcastle-Ottawa Scale.

Inverse-variance weighted random effects meta-analysis was used to derive overall summary estimates for all analyses. For studies that reported separate measures of relative risk for subgroups (for example, men and women), inverse-variance weighted fixed effects meta-analysis was used to derive a study-level relative risk prior to random effects meta-analysis. Heterogeneity was quantified using the $\mathrm{I}^{2}$ statistic and the Q-test. Publication bias was assessed using forest plots and Egger's test for coronary heart disease, cardiovascular mortality and stroke (the only outcomes reported in more than five studies).

Five sensitivity analyses were undertaken. First, studies were stratified by baseline year of assessment. Second, studies were stratified by length of follow-up. Third, studies were stratified by mean participant age. Tests for trend were performed 
for these continuous outcomes using meta-regression. Fourth, studies were stratified by the type of anxiety under investigation (generalized or unspecified anxiety, PTSD, panic disorder, phobic anxiety or worry), to examine whether different anxiety types differed in their association with cardiovascular disease. Fifth, studies were stratified by level of adjustment (minimally adjusted versus adequately adjusted), to examine whether further adjustment, as well as adjustment for potential mediators such as hypertension, attenuated the observed association between anxiety and cardiovascular disease. These five sensitivity analyses were again restricted to coronary heart disease, cardiovascular mortality and stroke, as other outcomes were reported in fewer than five studies.

\section{Results}

1804 studies were identified in the search and 1564 studies excluded in the abstract screen (Figure 1). After excluding an additional 194 studies in the full text screen, 46 studies were included in the meta-analysis. These 46 studies included 2017126 participants, in total, and 222253 participants with anxiety. Although we included chronic kidney disease as an outcome of interest in our protocol, no studies were identified that examined the association between anxiety and chronic kidney disease. Characteristics of included studies are provided in Table 1. Eleven studies were minimally adjusted while 35 studies were adequately adjusted (Supp. Table 1). Most studies were at low risk of bias when assessed using the Newcastle-Ottawa scale (Supp. Table 2).

Eight studies examined the association of anxiety with cardiovascular mortality. Anxiety was associated with a 41\% higher risk of cardiovascular mortality (RR 1.41 CI 1.13, 1.73; Figure 2 and Supp. Figure 1), with high heterogeneity among studies $\left(\mathrm{I}^{2}\right.$ $=65 \%$ ). Twenty-eight studies examined the association of anxiety with coronary heart disease. Anxiety was associated with a $41 \%$ higher risk of coronary heart disease (RR 
1.41 CI 1.23, 1.61; Figures 2 and 3). Heterogeneity among studies was high $\left(\mathrm{I}^{2}=96 \%\right)$. The strongest association observed was for stroke; anxiety was associated with a 71\% higher risk of stroke (RR 1.71 CI 1.18, 2.50; Figures 2 and 4), albeit with high heterogeneity $\left(I^{2}=90 \%\right)$. Anxiety was also associated with a 35\% higher risk of heart failure (RR 1.35 CI 1.11, 1.6; 4; $\mathrm{I}^{2}=57 \%$; Figure 2 and Supp. Figure 2) but was not significantly associated with major cardiovascular events (RR 1.64 CI 0.84, 3.19; Figure 2 and Supp. Figure 3) or atrial fibrillation (RR 1.27 CI 0.90, 1.80; Figure 2 and Supp. Figure 4).

No evidence of publication bias was observed for coronary heart disease or stroke (Supp. Figures 5 and 6). Evidence of publication bias was, however, observed for cardiovascular mortality ( $\mathrm{p}=0.018$, Supp. Figure 7). A trim-and-fill estimate for cardiovascular mortality was non-significantly elevated (RR 1.15 CI 0.92, 1.44).

For coronary heart disease, no evidence of an interaction was observed when studies were stratified by baseline year, period of follow up, mean age or level of adjustment in sensitivity analyses (Supp. Figure 8). However, phobic anxiety was more strongly associated with incident coronary heart disease than general/unspecified anxiety ( $\mathrm{p}$ value for the interaction $=0.004)$. For cardiovascular mortality, no evidence of an interaction was observed in sensitivity analyses (Supp. Figure 9). For stroke, stronger associations of anxiety were observed among adequately adjusted studies and among studies examining PTSD (Supp. Fig 10). Stronger associations were also observed among studies with later baseline year and studies with shorter follow up, although tests for trend were of borderline significance ( $p$ trend $>0.02$ ).

\section{Discussion}

In this meta-analysis, anxiety was associated with a $41 \%$ higher risk of cardiovascular mortality, a $41 \%$ higher risk of coronary heart disease, a $71 \%$ higher risk of stroke and a 35\% higher risk of heart failure. Although anxiety was not 
significantly associated with atrial fibrillation and major cardiovascular events, confidence intervals were wide and point estimates similar to other outcomes. Estimates were broadly similar in sensitivity analyses, although phobic anxiety was associated with a higher risk of coronary heart disease and PTSD associated with a higher risk of stroke than other anxiety types.

A large body of evidence has demonstrated that depression is a risk factor for cardiovascular disease. ${ }^{5,7}$ In a meta-analysis of 21 prospective studies, depression was associated with a $81 \%$ higher risk of coronary heart disease (RR 1.81 CI 1.53, 2.15). ${ }^{7}$ Our results suggest that, like depression, anxiety should be considered a risk factor for a wide range of cardiovascular diseases.

This meta-analysis extends prior work demonstrating anxiety to be associated with coronary heart disease. In a meta-analysis of twenty studies reporting on the association of anxiety and incident CHD, anxiety was associated with a $26 \%$ higher risk of CHD (HR 1.26 CI 1.15, 1.38). ${ }^{2}$ Our meta-analysis identified an additional eight studies and the pooled association of anxiety and coronary heart disease was similar (RR 1.41 CI 1.23, 1.61). However, we also observed strong associations between anxiety and cardiovascular mortality, heart failure and stroke. Indeed, the association of anxiety with stroke was stronger (RR 1.71 CI 1.18, 2.50) than that of the association of anxiety with coronary heart disease, although confidence intervals were wider. Although the association of anxiety with the composite endpoint of major cardiovascular events (cardiovascular death, non-fatal myocardial infarction, non-fatal stroke and non-fatal heart failure) was not significant, this is likely due to a lack of power, as only three studies were identified which reported on a cardiovascular composite and anxiety was independently associated with stroke, heart failure and coronary heart disease. Similarly, the lack of an association with atrial fibrillation was also likely due to a lack of power, as only three studies were identified that reported on 
the association between anxiety and atrial fibrillation, the effect estimate was similar to other outcomes and confidence intervals were wide. Larger studies examining the association between anxiety and atrial fibrillation could confirm this hypothesis.

Although there are several biological pathways by which anxiety may increase the risk of cardiovascular disease, it is unclear if the associations reported here are causal. However, even if the associations reported here are not causal, an elevated risk of cardiovascular disease among anxiety sufferers would support greater screening and more aggressive measures to prevent cardiovascular disease among individuals with anxiety.

This analysis has several strengths. We included more studies than any previous meta-analysis on anxiety and examined a wider range of outcomes. However, our analysis also has several limitations. First, we observed high levels of heterogeneity $\left(\mathrm{I}^{2}>50 \%\right)$ for all our outcomes, similar to prior meta-analyses of psychosocial risk factors for cardiovascular disease. ${ }^{2,8}$ These may be due to differences in the populations under study, such as types of anxiety under investigation and use of cardiovascular risk reducing therapies, as well as methodological characteristics, such as study design, length of follow-up and endpoint ascertainment. Second, we lacked individual patient data for studies, which would have allowed us to undertaken consistent adjustment for potential confounders. Third, studies which observed positive associations between anxiety and cardiovascular disease may have been more likely to be published. However, we did not observed evidence of publication bias for any outcomes other than cardiovascular mortality. 


\section{Funding}

Connor Emdin, Ayodele Odutayo, Jenny Tran and Benjamin Hunn are supported by the Rhodes Trust. Christopher Wong is supported by the Rhodes Trust and by a Neil Hamilton Fairly Fellowship from the National Health and Medical Research Council of Australia. Allan Hsiao is supported by a National Science Foundation Graduate Research Fellowship.

No funders or sponsors were involved in the design and conduct of the study; collection, management, analysis, and interpretation of the data; preparation, review, or approval of the manuscript; or decision to submit the manuscript for publication.

\section{Disclosures}

No conflicts of interests exist.

\section{Contributors}

CE had full access to all the data in the study and take responsibility for the integrity of the data and the accuracy of the data analysis. CE conceived of the study, acquired, analysed and interpreted the data, and drafted and critically revised the report. AO, JT, $\mathrm{BH}, \mathrm{AH}, \mathrm{CW}$ acquired, analysed and interpreted the data, and critically revised the report. 


\section{References}

1. Tsuchihashi-Makaya M, Kato N, Chishaki A, Takeshita A, Tsutsui H. Anxiety and poor social support are independently associated with adverse outcomes in patients with mild heart failure. Circ J. 2009;73:280-287.

2. Roest AM, Martens EJ, de Jonge P, Denollet J. Anxiety and risk of incident coronary heart disease: a meta-analysis. J Am Coll Cardiol. 2010;56:38-46.

3. Lambiase MJ, Kubzansky LD, Thurston RC. Prospective study of anxiety and incident stroke. Stroke. 2014;45:438-443.

4. Garfield LD, Scherrer JF, Hauptman PJ, Freedland KE, Chrusciel T, Balasubramanian S, Carney RM, Newcomer JW, Owen R, Bucholz KK, Lustman PJ. Association of anxiety disorders and depression with incident heart failure. Psychosom Med. 2014;76:128-136.

5. Phillips AC, Batty GD, Gale CR, Deary IJ, Osborn D, MacIntyre K, Carroll D. Generalized anxiety disorder, major depressive disorder, and their comorbidity as predictors of all-cause and cardiovascular mortality: the Vietnam experience study. Psychosom Med. 2009;71:395-403.

6. Denollet J, Maas K, Knottnerus A, Keyzer JJ, Pop VJ. Anxiety predicted premature all-cause and cardiovascular death in a 10-year follow-up of middleaged women. J Clin Epidemiol. 2009;62:452-456.

7. Nicholson A, Kuper H, Hemingway H. Depression as an aetiologic and prognostic factor in coronary heart disease: a meta-analysis of 6362 events among 146538 participants in 54 observational studies. Eur Heart J. 2006;27:2763-2774.

8. Pan A, Sun Q, Okereke OI, Rexrode KM, Hu FB. Depression and risk of stroke morbidity and mortality: a meta-analysis and systematic review. JAMA. 2011;306:1241-1249.

9. Berecki-Gisolf J, McKenzie SJ, Dobson AJ, McFarlane A, McLaughlin D. A history of comorbid depression and anxiety predicts new onset of heart disease. J Behav Med. 2013;36:347-353.

10. Boyle SH, Michalek JE, Suarez EC. Covariation of psychological attributes and incident coronary heart disease in U.S. Air Force veterans of the Vietnam war. Psychosom Med. 2006;68:844-850.

11. Butnoriene J, Bunevicius A, Saudargiene A, Nemeroff CB, Norkus A, Ciceniene V, Bunevicius R. Metabolic syndrome, major depression, generalized anxiety disorder, and ten-year all-cause and cardiovascular mortality in middle aged and elderly patients. Int J Cardiol. 2015;190:360-366.

12. Davidson KW, Mostofsky E, Whang W. Don't worry, be happy: positive affect and reduced 10-year incident coronary heart disease: the Canadian Nova Scotia Health Survey. Eur Heart J. 2010;31:1065-1070.

13. Eaker ED, Pinsky J, Castelli WP. Myocardial infarction and coronary death among women: psychosocial predictors from a 20-year follow-up of women in 
the Framingham Study. Am J Epidemiol. 1992;135:854-864.

14. Eaker ED, Sullivan LM, Kelly-Hayes M, D'Agostino RB, Benjamin EJ.

Tension and anxiety and the prediction of the 10-year incidence of coronary heart disease, atrial fibrillation, and total mortality: the Framingham Offspring Study. Psychosom Med. 2005;67:692-696.

15. Gustad LT, Laugsand LE, Janszky I, Dalen H, Bjerkeset O. Symptoms of anxiety and depression and risk of acute myocardial infarction: the HUNT 2 study. Eur Heart J. 2014;35:1394-1403.

16. Gustad LT, Laugsand LE, Janszky I, Dalen H, Bjerkeset O. Symptoms of anxiety and depression and risk of heart failure: the HUNT Study. Eur J Heart Fail. 2014;16:861-870.

17. Jakobsen AH, Foldager L, Parker G, Munk-Jørgensen P. Quantifying links between acute myocardial infarction and depression, anxiety and schizophrenia using case register databases. J Affect Disord. 2008;109:177-181.

18. Janszky I, Ahnve S, Lundberg I, Hemmingsson T. Early-onset depression, anxiety, and risk of subsequent coronary heart disease: 37-year follow-up of 49,321 young Swedish men. J Am Coll Cardiol. 2010;56:31-37.

19. Laan W, Termorshuizen F, Smeets HM, Boks MPM, de Wit NJ, Geerlings MI. A comorbid anxiety disorder does not result in an excess risk of death among patients with a depressive disorder. J Affect Disord. 2011;135:284-291.

20. Mykletun A, Bjerkeset O, Dewey M, Prince M, Overland S, Stewart R. Anxiety, depression, and cause-specific mortality: the HUNT study. Psychosom Med. 2007;69:323-331.

21. Nicholson A, Fuhrer R, Marmot M. Psychological distress as a predictor of CHD events in men: the effect of persistence and components of risk. Psychosom Med. 2005;67:522-530.

22. Ostir GV, Goodwin JS. High anxiety is associated with an increased risk of death in an older tri-ethnic population. J Clin Epidemiol. 2006;59:534-540.

23. Scherrer JF, Chrusciel T, Zeringue A, Garfield LD, Hauptman PJ, Lustman PJ, Freedland KE, Carney RM, Bucholz KK, Owen R, True WR. Anxiety disorders increase risk for incident myocardial infarction in depressed and nondepressed Veterans Administration patients. Am Heart J. 2010;159:772779 .

24. Seldenrijk A, Vogelzangs N, Batelaan NM, Wieman I, van Schaik DJF, Penninx BJWH. Depression, anxiety and 6-year risk of cardiovascular disease. J Psychosom Res. 2015;78:123-129.

25. Shirom A, Toker S, Jacobson O, Balicer RD. Feeling vigorous and the risks of all-cause mortality, ischemic heart disease, and diabetes: a 20-year follow-up of healthy employees. Psychosom Med. 2010;72:727-733.

26. Thurston RC, Kubzansky LD, Kawachi I, Berkman LF. Do depression and anxiety mediate the link between educational attainment and CHD? Psychosom 
Med. 2006;68:25-32.

27. Tolmunen T, Lehto SM, Julkunen J, Hintikka J, Kauhanen J. Trait anxiety and somatic concerns associate with increased mortality risk: a 23-year follow-up in aging men. Ann Epidemiol. 2014;24:463-468.

28. Ringbäck Weitoft G, Rosén M. Is perceived nervousness and anxiety a predictor of premature mortality and severe morbidity? A longitudinal follow up of the Swedish survey of living conditions. J Epidemiol Community Health. 2005;59:794-798.

29. Whang W, Davidson KW, Conen D, Tedrow UB, Everett BM, Albert CM. Global Psychological Distress and Risk of Atrial Fibrillation Among Women: The Women's Health Study. J Am Heart Assoc. 2012;1:e001107-e001107.

30. Yasuda N, Mino Y, Koda S, Ohara H. The differential influence of distinct clusters of psychiatric symptoms, as assessed by the general health questionnaire, on cause of death in older persons living in a rural community of Japan. J Am Geriatr Soc. 2002;50:313-320.

31. Batelaan NM, Have ten M, van Balkom AJLM, Tuithof M, de Graaf R. Anxiety disorders and onset of cardiovascular disease: the differential impact of panic, phobias and worry. J Anxiety Disord. 2014;28:252-258.

32. Chen Y-H, Tsai S-Y, Lee H-C, Lin H-C. Increased risk of acute myocardial infarction for patients with panic disorder: a nationwide population-based study. Psychosom Med. 2009;71:798-804.

33. Chen Y-H, Hu C-J, Lee H-C, Lin H-C. An increased risk of stroke among panic disorder patients: a 3-year follow-up study. Can J Psychiatry. 2010;55:43-49.

34. Cheng Y-F, Leu H-B, Su C-C, Huang C-C, Chiang C-H, Huang P-H, Chung CM, Lin S-J, Chen J-W, Chan W-L. Association between panic disorder and risk of atrial fibrillation:a nationwide study. Psychosom Med. 2013;75:30-35.

35. Gomez-Caminero A, Blumentals WA, Russo LJ, Brown RR, Castilla-Puentes R. Does panic disorder increase the risk of coronary heart disease? A cohort study of a national managed care database. Psychosom Med. 2005;67:688-691.

36. Smoller JW, Pollack MH, Wassertheil-Smoller S, Jackson RD, Oberman A, Wong ND, Sheps D. Panic attacks and risk of incident cardiovascular events among postmenopausal women in the Women's Health Initiative Observational Study. Arch Gen Psychiatry. 2007;64:1153-1160.

37. Walters K, Rait G, Petersen I, Williams R, Nazareth I. Panic disorder and risk of new onset coronary heart disease, acute myocardial infarction, and cardiac mortality: cohort study using the general practice research database. Eur Heart J. 2008;29:2981-2988.

38. Albert CM, Chae CU, Rexrode KM, Manson JE, Kawachi I. Phobic anxiety and risk of coronary heart disease and sudden cardiac death among women. Circulation. 2005;111:480-487. 
39. Haines AP, Imeson JD, Meade TW. Phobic anxiety and ischaemic heart disease. Br Med J (Clin Res Ed). 1987;295:297-299.

40. Kawachi I, Colditz GA, Ascherio A, Rimm EB, Giovannucci E, Stampfer MJ, Willett WC. Prospective study of phobic anxiety and risk of coronary heart disease in men. Circulation. 1994;89:1992-1997.

41. Boscarino JA. Posttraumatic stress disorder and mortality among U.S. Army veterans 30 years after military service. Ann Epidemiol. 2006;16:248-256.

42. Boscarino JA. A prospective study of PTSD and early-age heart disease mortality among Vietnam veterans: implications for surveillance and prevention. Psychosom Med. 2008;70:668-676.

43. Chen M-H, Pan T-L, Li C-T, Lin W-C, Chen Y-S, Lee Y-C, Tsai S-J, Hsu J-W, Huang K-L, Tsai C-F, Chang W-H, Chen T-J, Su T-P, Bai Y-M. Risk of stroke among patients with post-traumatic stress disorder: nationwide longitudinal study. Br J Psychiatry. 2015;206:302-307.

44. Crum-Cianflone NF, Bagnell ME, Schaller E, Boyko EJ, Smith B, Maynard C, Ulmer CS, Vernalis M, Smith TC. Impact of combat deployment and posttraumatic stress disorder on newly reported coronary heart disease among US active duty and reserve forces. Circulation. 2014;129:1813-1820.

45. Jordan HT, Stellman SD, Morabia A, Miller-Archie SA, Alper H, Laskaris Z, Brackbill RM, Cone JE. Cardiovascular disease hospitalizations in relation to exposure to the September 11, 2001 World Trade Center disaster and posttraumatic stress disorder. J Am Heart Assoc. 2013;2:e000431-e000431.

46. Kubzansky LD, Koenen KC, Spiro A, Vokonas PS, Sparrow D. Prospective study of posttraumatic stress disorder symptoms and coronary heart disease in the Normative Aging Study. Arch Gen Psychiatry. 2007;64:109-116.

47. Kubzansky LD, Koenen KC, Jones C, Eaton WW. A prospective study of posttraumatic stress disorder symptoms and coronary heart disease in women. Health Psychol. 2009;28:125-130.

48. Roy SS, Foraker RE, Girton RA, Mansfield AJ. Posttraumatic stress disorder and incident heart failure among a community-based sample of US veterans. Am J Public Health. 2015;105:757-763.

49. Vaccarino V, Goldberg J, Rooks C, Shah AJ, Veledar E, Faber TL, Votaw JR, Forsberg CW, Bremner JD. Post-traumatic stress disorder and incidence of coronary heart disease: a twin study. J Am Coll Cardiol. 2013;62:970-978.

50. Vogt T, Pope C, Mullooly J, Hollis J. Mental health status as a predictor of morbidity and mortality: a 15-year follow-up of members of a health maintenance organization. Am J Public Health. 1994;84:227-231. 


\section{Figures}

Figure 1. Identification of studies.

Figure 2. Association of anxiety with cardiovascular disease.

Figure 3. Association of anxiety with coronary heart disease.

Figure 4. Association of anxiety with stroke. 